\title{
Perfect-Information Stochastic Parity Games
}

\author{
Wiesław Zielonka \\ LIAFA, case 7014 \\ Université Paris 7 \\ 2, Place Jussieu \\ 75251 Paris Cedex 05, France \\ zielonka@liafa.jussieu.fr
}

\begin{abstract}
We show that in perfect-information stochastic parity games with a finite state space both players have optimal pure positional strategies. Contrary to the recent proofs of this fact by K. Chatterejee, M. Jurdziński, T.A. Henzinger 2 and A.K. McIver, C.C. Morgan 14 the proof given in this paper proceeds by a straightforward induction on the number of outgoing transitions available to one of the players and is self-contained.
\end{abstract}

\section{Introduction}

The subject of infinite stochastic games takes its source in the paper of Shapley 15. where the existence of value is proved for discounted games. In the sequel other types of payments for repetitive games, like mean payoff, traditionally examined by game theory community were also applied in the context of stochastic games, see [11 for the comprehensive textbook treatment of the subject. More recently stochastic games entered in the domain of computer science where the main motivation is in the verification of properties of probabilistic programs [7, 8,5 14. However, in this new context the natural winning criterion is given by the parity condition.

A perfect-information stochastic parity game is played by two players on a graph with the vertices partitioned on three sets $S_{0}, S_{1}$ and $S_{p}$, where $S_{0}$ and $S_{p}$ are the vertices of players Even and Odd while $S_{p}$ is the set of randomized vertices. The players play by moving the token between vertices, if the token is in a vertex $s \in S_{0} \cup S_{1}$ then the corresponding player moves it to some successor vertex. With each randomized vertex $s \in S_{p}$ there is associated a fixed probability distribution of successor vertices; if the token visits such a vertex then the next position is chosen according to this distribution. The winning player is determined by the parity condition: the vertices are labeled with non negative integers - priorities - and if the maximal priority visited infinitely often during an infinite play is even then the player Even wins, otherwise the player Odd wins.

^ This research was supported by European Research Training Network: Games and Automata for Synthesis and Validation and Action Spécifique: Automates, modèles distribués et temporisés 
The objective of each player to maximize his winning probability. The main result of the present paper is that each player has an optimal positional (memoryless) and pure (deterministic) strategy for switching control parity games over finite state space. Since the number of possible positional pure strategies is exponential in the number of edges, this allows to compute optimal strategies and maximal winning probabilities in exponential time. Since one player case can be solved polynomially two-player parity games are in fact in NP^co-NP [2]. Note that for simpler reachability objectives the existence of optimal pure positional strategies was proved in 3 .

The results presented here do not carry over to an infinite state space, there exist infinite simple stochastic Büchi games with just one player such that this player has only non positional $\epsilon$-optimal strategies 12 .

Let us note that the existence of pure positional strategies is known for other games such as non stochastic parity games (also with infinite state space) [9] or discounted and average stochastic perfect information games [1].

This contrasts with more complex stochastic games with simultaneous moves which were examined in a series of papers 7 , 5,6]. For such games the game value can be non rational even if the data are rational and only $\epsilon$-optimal infinite memory strategies are in general available. The main achievement in this direction is the proof of the existence of the value for "concurrent" stochastic parity games [7]. It is interesting to note that while "deterministic" parity games are closely related to model checking and $\mu$ calculus [1,10] over finite lattices, [7] shows that concurrent stochastic parity games are related to $\mu$-calculus over infinite lattices of mappings from the set state to the interval $[0 ; 1]$.

Recently K. Chatterejee, M. Jurdziński, T.A. Henzinger [2] have given another proof of the existence of memoryless pure optimal strategies in switching control parity games. However, their proof is quite different, in particular they rely on the non trivial result concerning the existence of the value in stochastic games with simultaneous moves 713.

Our approach of the problem is more elementary, we prove at the same time the existence of the value and the existence of optimal memoryless pure strategies by induction on the number of edges. The technique developed here for parity games can be adapted to other situations where the game arena is finite.

\section{Preliminaries}

Simple parity games are played by two players Even and Odd on arenas $\left(S_{0}, S_{1}, S_{p}\right.$, succ, $p, g$ ) consisting of the following elements: $S_{0}, S_{1}, S_{p}$ are three disjoint sets of states, the states $S_{0}$ and $S_{1}$ belong respectively to the player Even and the player Odd while $S_{p}$ is the set of randomized states, $g: S \longrightarrow[0 . . n]$ is a priority mapping from the set $S=S_{0} \cup S_{1} \cup S_{p}$ of all states to an initial segment $[0 . . n]$ of non negative integers $(g(s)$ is the priority of the state $s)$, succ : $S_{0} \cup S_{1} \longrightarrow \mathcal{P}(S)$ is the successor mapping associating with each state of $S_{0} \cup S_{1}$ a non empty set of available successor states. Finally, $p$ is a set of conditional probabilities: for each randomized state $s \in S_{p}$ and any state $s^{\prime} \in S$, 
$p\left(s^{\prime} \mid s\right)$ is the probability that the next state is $s^{\prime}$ under the condition that the current state is $s$. It is convenient to extend the successor mapping to randomized states by setting for $s \in S_{p}, \operatorname{succ}(s)=\left\{s^{\prime} \mid p\left(s^{\prime} \mid s\right)>0\right\}$. We assume that $p$ satisfies the usual conditions of probability distribution:

$$
\text { for all } s \in S_{p}, \sum_{s^{\prime} \in \operatorname{succ}(s)} p\left(s^{\prime} \mid s\right)=1 \text {, where } 0 \leq p\left(s^{\prime} \mid s\right) \leq 1 \text {. }
$$

Given an arena, the parity game $G(s)$ is played in the following way. The initial state at the moment 0 is $s=s_{0}$. For all moments $i \geq 0$, if the current state is $s_{i} \in S$ then

(1) if $s_{i} \in S_{0}$ then player Even chooses the next state $s_{i+1} \in \operatorname{succ}\left(s_{i}\right)$,

(2) if $s_{i} \in S_{1}$ then player Odd chooses the next state $s_{i+1} \in \operatorname{succ}\left(s_{i}\right)$,

(3) if $s_{i} \in S_{p}$ then the next state $s_{i+1}$ is chosen with the probability $p\left(s_{i+1} \mid s_{i}\right)$.

Thus after an infinite number of steps we get an infinite sequence $\left(s_{i}\right)_{i=0}^{\infty}$ of visited states that is called a play in the game $G(s)$.

Let $\limsup _{i} g\left(s_{i}\right)$ be the maximal priority visited infinitely often in the play $\left(s_{i}\right)_{i=0}^{\infty}$. If this priority is even then the player Even wins the play, otherwise the player Odd wins.

We have assumed here that for each state $s \in S, \operatorname{succ}(s) \neq \emptyset$. The case of terminating plays with $\operatorname{succ}(s)=\emptyset$ for some states $s$ can be easily adapted by introducing for each such state a self-loop by setting $\operatorname{succ}(s)=\{s\}$.

In the sequel by $\operatorname{Win}_{0}(s)$ and $\operatorname{Win}_{1}(s)$ we shall note respectively the set of winning plays for the players Even and Odd in the game $G(s)$, i.e. plays where the maximal priority visited infinitely often is even/odd.

Informally, the aim of each player is to maximize his probability of winning the parity game.

Any non empty finite sequence of states starting from $s$ is a history in $G(s)$.

A strategy $\sigma$ for the player Even in $G\left(s_{0}\right)$ is a mapping assigning to each history $s_{0} s_{1} \ldots s_{n}$ starting at $s_{0}$ and terminating at a state $s_{n} \in S_{0}$ the conditional probability $\sigma\left(\cdot \mid s_{0} s_{1} \ldots s_{n}\right)$ of choosing the next state. We assume that only the states available at the current state $s_{n}$ can be chosen, i.e. $\sum_{s^{\prime} \in \operatorname{succ}\left(s_{n}\right)} \sigma\left(s^{\prime} \mid s_{0} s_{1} \ldots s_{n}\right)=1$ and $\sigma\left(s^{\prime} \mid s_{0} s_{1} \ldots s_{n}\right)=0$ for $s^{\prime} \notin \operatorname{succ}\left(s_{n}\right)$.

A strategy $\tau$ for the player Odd is defined symmetrically.

In the sequel, $\sigma$ and $\tau$, with subscripts if necessary, will always stand for strategies of Even and Odd.

Given the strategies $\sigma$ and $\tau$ of Even and Odd in $G\left(s_{0}\right)$ we can draw a probability tree: the histories $s_{0} S^{*}$ constitute its vertices and we assign the probability $\alpha\left(s_{n+1} \mid s_{0} s_{1} \ldots s_{n}\right)$ to the edge going from the vertex $s_{0} s_{1} \ldots s_{n}$ to the vertex $s_{0} s_{1} \ldots s_{n} s_{n+1}$, where $\alpha$ is either $\sigma$, or $\tau$, or $p$ depending on whether the last state $s_{n}$ in the history belongs to $S_{0} S_{1}$, or $S_{p}$. Then the probability of an open set $s_{0} s_{1} \ldots s_{k} S^{\omega}$, is obtained by multiplying all probabilities along the path from $s_{0}$ to $s_{0} s_{1} \ldots s_{k}$ in the probability tree. It is a standard result of measure theory (uniqueness of bounded measures defined with $\pi$-systems) that this extends to a unique probability measure over Borel sets of plays starting from $s_{0}$. This probability measure will be noted by $P_{\sigma, \tau}$.

A parity game $G(s)$ in the normal form is played in the following way: the player Even chooses a strategy $\sigma$ and in the same time and independently 
the player Odd chooses a strategy $\tau$. Then Even receives $P_{\sigma, \tau}\left(\operatorname{Win}_{0}(s)\right)$ while Odd receives $P_{\sigma, \tau}\left(\operatorname{Win}_{1}(s)\right)$ and the aim of each player is to maximize his gain. Since $P_{\sigma, \tau}\left(\operatorname{Win}_{1}(s)\right)+P_{\sigma, \tau}\left(\operatorname{Win}_{0}(s)\right)=1$ this is a constant sum game. (We can formulate it equivalently as a zero sum game if we assume that Odd pays to Even the amount $P_{\sigma, \tau}\left(\operatorname{Win}_{0}(s)\right)$, then the aim of Odd would be to minimize $P_{\sigma, \tau}\left(\operatorname{Win}_{0}(s)\right)$. We prefer however the first formulation since it allows more a symmetrical treatment of both players.)

We have always

$$
\begin{aligned}
& \underline{\operatorname{val}}_{0}(G(s))=\sup _{\sigma} \inf _{\tau} P_{\sigma, \tau}\left(\operatorname{Win}_{0}(s)\right) \leq \inf _{\tau} \sup _{\sigma} P_{\sigma, \tau}\left(\operatorname{Win}_{0}(s)\right)=\overline{\operatorname{val}}_{0}(G(s)) \\
& \underline{\operatorname{val}}_{1}(G(s))=\sup _{\tau} \inf _{\sigma} P_{\sigma, \tau}\left(\operatorname{Win}_{1}(s)\right) \leq \inf _{\sigma} \sup _{\tau} P_{\sigma, \tau}\left(\operatorname{Win}_{1}(s)\right)=\overline{\operatorname{val}}_{1}(G(s))
\end{aligned}
$$

Notation. In the sequel, it will be sometimes convenient to use 0 and 1 as synonyms of the names Even and Odd of players.

The quantities $\underline{\mathrm{val}}_{i}(G(s))$ and $\overline{\mathrm{val}}_{i}(G(s))$ are called respectively the lower and upper values of the game $G(s)$ for the player $i$. If the lower and upper values are equal then this quantity is called the value of the game $G(s)$ (for the player $i$ ) and is noted as $\operatorname{val}_{i}(G(s))$.

Note the obvious equalities: $\underline{\operatorname{val}}_{0}(G(s))=1-\overline{\operatorname{val}}_{1}(G(s))$ and $\overline{\operatorname{val}}_{0}(G(s))=$ $1-\underline{\operatorname{val}}_{1}(G(s))$, in particular $\operatorname{val}_{1}(G(s))=1-\operatorname{val}_{0}(G(s))$ where the left hand side exists iff the right hand side exists.

A strategy $\bar{\sigma}$ of the player Even in $G(s)$ is optimal if the value $\operatorname{val}_{0}(G(s))$ exists and, for all strategies $\tau$ of Odd, $P_{\bar{\sigma}, \tau}\left(\operatorname{Win}_{0}(s)\right) \geq \operatorname{val}_{0}(G(s))$. Symmetrically, $\bar{\tau}$ is optimal for Odd if the game value exists and, for all strategies $\sigma$ of Even $P_{\sigma, \bar{\tau}}\left(\operatorname{Win}_{1}(s)\right) \geq \operatorname{val}_{1}(G(s))$ or equivalently $P_{\sigma, \bar{\tau}}\left(\operatorname{Win}_{0}(s)\right) \leq \operatorname{val}_{0}(G(s))$.

Among all available strategies, the simplest are positional and pure strategies.

A strategy $\sigma$ for player Even is positional if the probability $\sigma\left(s_{n+1}\right.$ | $\left.s_{0} \ldots s_{n}\right), s_{n} \in S_{0}$, depends only on the current state $s_{n}$ and is independent of the preceding history. Thus such a strategy can be seen as a family of conditional probabilities $\sigma(\cdot \mid s), s \in S_{0}$, satisfying the usual conditions: $\sum_{s^{\prime} \in \operatorname{succ}(s)} \sigma\left(s^{\prime} \mid s\right)=1$ and $\sigma\left(s^{\prime} \mid s\right)=0$ for $s^{\prime} \notin \operatorname{succ}(s)$

A strategy $\sigma$ is pure if it choses exactly one of the successor states with probability one.

A strategy $\sigma$ for player Even is universally optimal if $\sigma$ is positional pure and optimal for all games $G(s)$, i.e. optimal independently of the initial state.

The notions of positional, pure and universally optimal strategies for Odd player are defined symmetrically.

The aim of this paper is to prove the following result:

Theorem 1. If the set of states is finite then for each initial state the parity game $G(s)$ has a value. Moreover, each player has an universally optimal strategy.

In the sequel we always assume that the set of states is always finite. 


\section{One Player Stochastic Parity Games}

A Markov decision process (MDP) with the parity criterion is a simple parity game where one of the two players is dummy, i.e. his set of states is empty. All the information about parity MDP useful in the sequel can be deduced from [8] and [4, we recall here these results for the sake of completeness and apply them in the context of parity MDP.

Suppose that $G=\left(S_{1}, S_{p}\right.$, succ, $\left.p, g\right)$ is a parity MDP, where the set of states of player Even is empty (the case where rather $S_{1}$ is empty can be treated in the same way).

Such games have always a value and the aim of this section is to prove that Odd has an universally optimal strategy $\tau$ and to examine in detail the structure of $\tau$.

With MPD $G$ we can associate the graph, noted $\mathbf{G}$, of $G$ : the vertices of this graph are the states of $G$ and the edges are pairs $\left(s, s^{\prime}\right)$ such that $s^{\prime} \in \operatorname{succ}(s)$.

The following notion appears in $[8$, although similar concept appears also in [4.

An end component of $G$ is a subgraph $\mathbf{G}^{\prime}$ of $\mathbf{G}$ such that: (1) for each randomized state of $\mathbf{G}^{\prime}$, the graph $\mathbf{G}^{\prime}$ contains also all successor states $s^{\prime}$ of $s$ together with all corresponding edges $\left(s, s^{\prime}\right),(2)$ for all non randomized states $s$ of $\mathbf{G}^{\prime}, \mathbf{G}^{\prime}$ contains at least one outgoing edge (together with the corresponding successor state) and (3) $\mathbf{G}^{\prime}$ is strongly connected.

Lemma $1([\mathbf{8}])$. With each infinite play $\mathbf{s}=\left(s_{i}\right)_{i=0}^{\infty}$ in $G$ starting from $s=s_{0}$ we associate a subgraph $\inf (\mathbf{s})$ of $\mathbf{G}$ consisting of the states and edges that appear infinitely often in $\mathbf{s}$. For any strategy $\tau$ of Odd in $M D P G(s)$, the set of plays $\mathbf{s}$ such that $\inf (\mathbf{s})$ is an end component of $\mathbf{G}$ has measure 1.

Proof. If a non randomized state $s$ is visited infinitely often in $\mathbf{s}$ then for at least one of its successors $s^{\prime}$ the edge $\left(s, s^{\prime}\right)$ is visited infinitely often.

If a randomized state $s$ is visited infinitely often then almost always (i.e. with probability one) all outgoing edges $\left(s, s^{\prime}\right)$ are visited infinitely often. (More exactly, for any strategy $\tau$, the set of plays where $s$ appears infinitely often has the same measure as the set of plays where all outgoing edges $\left(s, s^{\prime}\right)$ appear infinitely often ). These two facts yield the thesis.

Lemma 2. Suppose that the greatest priority inside of an end component $\mathbf{G}^{\prime}$ is odd. Let $U$ be the set of states of $\mathbf{G}^{\prime}$. Then the player $\mathbf{O d d}$ has a positional pure strategy on $U$ allowing him to visit the states with the maximal priority infinitely often with probability 1 (and he wins in this way the parity game with probability 1 as well). Moreover, as long as he uses this strategy the token never leaves $U$.

Proof. Let $\mathbf{G}^{\prime}=(U, E)$ and $A$ the subset of $U$ consisting of vertices with the maximal priority. We define the distance of a vertex $s$ of $U$ from $A$ as the length of the shortest path in $\mathbf{G}^{\prime}$ from $s$ to some vertex of $A$. Thus any vertex $s \in U$ at the distance $i>0$ has at least one successor at the distance $i-1$. Now the 
strategy of player Odd consists in choosing always a fixed successor with the smallest distance from $A$. Suppose that using this strategy we do not visit $A$ infinitely often and that $s$ is a vertex visited infinitely often with the smallest distance from $A$. However if $s$ belongs to Odd then, with the strategy described above, some successor of $s$ is visited infinitely often. If $s$ is a randomized vertex then with probability one all outgoing edges, and therefore all successor vertices are visited infinitely often, in particular the one closer to $A$ than $s$ itself. Finally note that the moves at the random states of $U$ can never put the token outside of $U$ (since all successors of such states are in $U$ ) and the same holds for the moves in the non random states advised by the strategy described above.

Let us call an end component $\mathbf{G}^{\prime}$ favorable for the player Odd if it satisfies the conditions of Lemma 2 , i.e. the maximal priority in $\mathbf{G}^{\prime}$ is odd. A state $s$ is said to be favorable (for the player Odd) if it belongs to some favorable end component.

Lemma 3. Let $U$ be the set of all favorable states. Player Odd has a positional pure strategy over $U$ allowing him to win the parity game with the probability 1.

Proof. Let us fix an enumeration of all favorable end components and let $U_{i}$ be the set of states of the $i$-th end component in this enumeration. Thus $U=\bigcup_{i} U_{i}$.

By Lemma 2 on each $U_{i}$ the player Odd has a positional pure strategy $\tau_{i}$ allowing him to win with the probability 1 . Let $s \in U \cap S_{1}$ and $l=\min \{i \mid s \in$ $U_{i}$ \}. The when the token is in $s$ the player Odd applies the strategy $\tau_{l}$.

In this way, for each play, Odd will change only finitely many times his strategy, with each change taking a new strategy $\tau_{i}$ with a smaller index $i$. From some moment onward, the remaining play will be played with a fixed strategy $\tau_{i}$ and therefore Odd will win it with the probability 1. (The formal reasoning will use the residual strategies and the decomposition defined at the beginning of the next section.)

Proposition 1. Player Odd has an universally optimal strategy $\bar{\tau}$ in parity $M D P G=\left(S_{1}, S_{p}\right.$, succ, $\left.p, g\right)$. Let $U$ be the set of favorable states for player Odd in $G$. The strategy $\bar{\tau}$ consists in attracting the token to $U$ with the maximal probability and next in playing the optimal strategy in $U$.

Let $X$ be the set of states s such that there is no path in the graph $\mathbf{G}$ of $G$ from $s$ to $U$. In all games $G(s), s \in X$, player Odd loses with probability 1 for all his strategies $\tau$.

If player Odd uses his universally optimal strategy $\bar{\tau}$ described above then with probability 1 the token hits either $U$ or $X$ and once in one of these two sets it will never leave it.

If $s \in S \backslash U$ then for any strategy $\tau$ of Odd in $G(s)$ if the token does not enter $U$ then player Odd loses with probability 1 , more formally $P_{\tau}\left(\operatorname{Win}_{1}(s) \cap\right.$ $\left.s(S \backslash U)^{\omega}\right)=0$ for $s \in S \backslash U$.

Proof. Suppose that $s \in S_{1} \backslash U$, where $U$ is the set of all favorable states for the player Odd and let $\tau$ be his strategy in $G(s)$. As we know from Lemma 1 
with probability 1 the set of states and edges that appear infinitely often when Odd plays $\tau$ forms and end component. If such a component does not intersect $U$ then, by the definition of $U$ the play ending in this end component is lost for the player Odd. Therefore the optimal strategy for Odd consists in fact in attracting the token to $U$ with the maximal probability and next play in $U$ using his positional pure strategy as shown in Lemma 3. The problem of finding the maximal probability to reach a fixed set of states can be formulated as a linear programming problem and moreover it can be shown that the player Odd has an optimal positional pure strategy assuring him the maximal probability to reach $U$, see $4,3,8$.

Now suppose that the play starts in a state $s \in X$. Then the token never reaches the set $U$ and therefore the probability to win the parity game $G(s)$ for $s \in X$ is 0 for any possible strategy of Odd. On the other hand, if the state $s$ is such that there exists in $\mathbf{G}$ a path from $s$ to $U$ then obviously there is a strategy to reach $U$ with a positive probability, and therefore a strategy to win the parity game $G(s)$ with a positive probability.

Thus $X$ is precisely the set of states where Odd loses almost surely.

\section{Two Player Stochastic Parity Games}

Two simplify the notation, we shall assume that each state of Odd at most two successors.

We shall prove that the parity games on finite arenas have values for all initial states and that player Even has an optimal positional pure strategy by induction on the number of successors available at states of $S_{0}$.

If for each state $s \in S_{0}, \operatorname{succ}(s)$ contains exactly one state then payer Even is in fact dummy and basically we have one player stochastic parity game controlled completely by player Odd. Then, as explained in Section 3 , the game has a value and player Odd has a positional pure optimal strategy. Since player Even has always one successor state for each $s \in S_{0}$ he has a unique strategy available for him which is positional and pure (and optimal as he has no alternative).

We shall use the following notation for games $G$ that have a value for each starting state $s$ :

$$
\begin{aligned}
& \mathrm{V}_{i}^{=1}(G)=\left\{s \in S \mid \operatorname{val}_{i}(G(s))=1\right\} \\
& \mathrm{V}_{i}^{>0}(G)=\left\{s \in S \mid \operatorname{val}_{i}(G(s))>0\right\}
\end{aligned}
$$

denote respectively the set of states where the player $i$ can win with the probability 1 and the set of states where he can win with a (strictly) positive probability.

Let $G$ be a parity game, $h=s_{0} \ldots s_{k}$ a history starting at $s_{0}$ and ending at some state $s_{k}$ and $\alpha$ a strategy for a player $i$ in $G\left(s_{0}\right)$. The residual strategy $\alpha_{h}$ for the player $i$ is the strategy in the game $G\left(s_{k}\right)$ defined as $\alpha_{h}\left(s_{k} s_{k+1} \ldots s_{n}\right)=$ $\alpha\left(h s_{k+1} \ldots s_{n}\right)$, where $s_{k} s_{k+1} \ldots s_{n}$ is a history starting at $s_{k}$ and ending in a state $s_{n} \in S_{i}$. 
A strategy $\tau$ of the player Odd in the game $G(s)$ is residually optimal if for all histories $h$ starting in $s$ and ending in a state $s^{\prime} \in S_{1}$, the residual strategy $\sigma_{h}$ is optimal in the game $G\left(s^{\prime}\right)$.

Definition 1. Suppose that $G$ is a parity game such that the value exists for all states. Let $U$ be a set of states and $s \in U$. A strategy $\tau$ for player Odd in the game $G(s)$ is persistent over $U$ if for any strategy $\sigma$ of Even in $G(s)$, $P_{\sigma, \tau}\left(\operatorname{Win}_{1}(s) \cap s U^{\omega}\right)=P_{\sigma, \tau}\left(s U^{\omega}\right)$.

Thus if the player Odd plays using a persistent strategy over $U$ and if the token never leaves $U$ then Odd wins almost surely.

Since several lemmas below share the same conditions we shall list them now for the sake of convenience.

Condition A. $\left(S_{0}, S_{1}, S_{p}\right.$, succ, $\left.p, g\right)$ is an arena of a game $G$ and $s \in S_{0}$ a state of player Even with two successors, $\operatorname{succ}(s)=\left\{s_{1}, s_{2}\right\}$. By $G_{i}, i=1,2$, we denote the parity games on arenas $\left(S_{0}, S_{1}, S_{p}, \operatorname{succ}_{i}, p, g\right)$ that are identical with $G$, except that in $G_{i}$ the state $s$ has only one $\operatorname{successor} \operatorname{succ}_{i}(s)=\left\{s_{i}\right\}$.

Condition B. The games $G_{1}$ and $G_{2}$ have values for all initial states $s$.

Condition C. Player Even has optimal positional pure strategies $\bar{\sigma}_{1}$ and $\bar{\sigma}_{2}$ in the games $G_{1}$ and $G_{2}$ respectively; these strategies are universally optimal, i.e. optimal independently of the starting state.

Condition D. For each state $s^{\prime} \in S$, player Odd has residually optimal strategies $\bar{\tau}_{1}$ and $\bar{\tau}_{2}$ in the games $G_{1}\left(s^{\prime}\right)$ and $G_{2}\left(s^{\prime}\right)$ respectively. Moreover, each $\bar{\tau}_{i}, i=1,2$, is persistent over $\mathrm{V}_{1}^{>0}\left(G_{i}\right)$.

Proposition 2. Suppose that Conditions A, B, $C$ and $D$ are satisfied and $\operatorname{val}_{0}\left(G_{2}(s)\right) \leq \operatorname{val}_{0}\left(G_{1}(s)\right)$ (the case $\operatorname{val}_{0}\left(G_{1}(s)\right) \leq \operatorname{val}_{0}\left(G_{2}(s)\right.$ ) is symmetrical). Then the following assertions hold:

(i) For all states $s^{\prime} \in S$, $\operatorname{val}_{0}\left(G_{2}\left(s^{\prime}\right)\right) \leq \operatorname{val}_{0}\left(G_{1}\left(s^{\prime}\right)\right)$ and $\operatorname{val}_{1}\left(G_{1}\left(s^{\prime}\right)\right) \leq$ $\operatorname{val}_{1}\left(G_{2}\left(s^{\prime}\right)\right)$, in particular $\mathrm{V}_{0}^{>0}\left(G_{2}\right) \subseteq \mathrm{V}_{0}^{>0}\left(G_{1}\right)$ and $\mathrm{V}_{1}^{>0}\left(G_{1}\right) \subseteq \mathrm{V}_{1}^{>0}\left(G_{2}\right)$.

(ii) $G$ has the same values as $G_{1}$, i.e. for $s^{\prime} \in S$, $\operatorname{val}_{0}\left(G\left(s^{\prime}\right)\right)=\operatorname{val}_{0}\left(G_{1}\left(s^{\prime}\right)\right)$.

(iii) The strategy $\bar{\sigma}_{1}$ optimal in $G_{1}$ for Even is also optimal for him in $G$, for $s^{\prime} \in S$ it assures him the gain of at least $\operatorname{val}_{0}\left(G_{1}\left(s^{\prime}\right)\right)$, i.e.

$\inf _{\tau} P_{\bar{\sigma}_{1}, \tau}\left(\operatorname{Win}_{0}\left(s^{\prime}\right)\right) \geq \operatorname{val}_{0}\left(G_{1}\left(s^{\prime}\right)\right)$, where $\tau$ ranges over all strategies of Odd in the game $G\left(s^{\prime}\right)$.

(iv) For each starting state $s^{\prime} \in S$, there exists a strategy $\bar{\tau}$ of the player Odd in the game $G\left(s^{\prime}\right)$ such that

(1) $\bar{\tau}$ is optimal, it assures that Even cannot win more than $\operatorname{val}_{0}\left(G_{1}\left(s^{\prime}\right)\right)$, i.e. $\sup _{\sigma} P_{\sigma, \bar{\tau}}\left(\operatorname{Win}_{0}\left(s^{\prime}\right)\right) \leq \operatorname{val}_{0}\left(G_{1}\left(s^{\prime}\right)\right)$, where $\sigma$ ranges over the strategies of Even in the game $G\left(s^{\prime}\right)$.

(2) $\bar{\tau}$ is residually optimal,

(3) $\bar{\tau}$ is persistent over the set $\mathrm{V}_{1}^{>0}\left(G_{1}\right)$.

Let us note the asymmetry in the treatment of the players Even and Odd in Proposition 2, While at each stage of the induction the optimal strategies of Even are always pure and positional, this property does not hold for the 
strategies of Odd. In fact, the optimal strategies of Odd in $G$ will be obtained from optimal strategies of Odd in $G_{1}$ and $G_{2}$ in such a way that the resulting strategy will be not positional in general (but always it will be pure).

We can apply immediately Proposition 2 to get:

\section{Proof of Theorem [1]}

As we have seen in Proposition 1 the unique player in one person parity games has an optimal positional pure strategy $\bar{\tau}$. Let $\mathrm{V}_{1}^{>0}(G)$ be the set of states where his winning probability is positive. If the game starts in $\mathrm{V}_{1}^{>0}(G)$ and the unique player uses $\bar{\tau}$ then with probability 1 the token either hits the set of states where he wins with probability 1 or it hits the set of states where the player wins with probability 0 . In particular, when Odd plays $\bar{\tau}$ and the token remains forever in $\mathrm{V}_{1}^{>0}(G)$ then in fact the token hits $\mathrm{V}_{1}^{=1}(G)$ with probability 1 and hence he wins with probability 1 . Thus $\bar{\tau}$ is trivially persistent over $\mathrm{V}_{1}^{>0}(G)$. Therefore Conditions A - D are satisfied for the games where the player Even is dummy.

Proposition 2] shows that this situation carries over when we use the induction over the number of successor states for player Even. Suppose that A - D hold. If we have solved the games $G_{1}$ and $G_{2}$ then it suffices to compare the winning probabilities at the state $s$ to choose the optimal strategy for Even: if $\operatorname{val}_{0}\left(G_{1}(s)\right) \geq \operatorname{val}_{0}\left(G_{2}(s)\right)$ then the universally optimal strategy $\bar{\sigma}_{1}$ in $G_{1}$ is also universally optimal in $G$ otherwise the strategy $\bar{\sigma}_{2}$ universally optimal in $G_{2}$ is universally optimal in $G$. In fact this choice allows the player Even to win at least $\max \left\{\operatorname{val}_{0}\left(G_{1}\left(s^{\prime}\right)\right), \operatorname{val}_{0}\left(G_{2}\left(s^{\prime}\right)\right)\right\}$ in $G\left(s^{\prime}\right)$. To show that he cannot win more, it is necessary to construct a strategy $\bar{\tau}$ of Odd in $G\left(s^{\prime}\right)$ such that playing against $\bar{\tau}$ the player Even wins at most $\max \left\{\operatorname{val}_{0}\left(G_{1}\left(s^{\prime}\right)\right), \operatorname{val}_{0}\left(G_{2}\left(s^{\prime}\right)\right)\right\}$, this is the last part of Proposition 2 .

Thus by induction we establish that Even has always an optimal pure positional strategy. Of course, exchanging the roles of Even and Odd we can establish the existence of an optimal pure positional strategy for Odd.

Let $X \subseteq s S^{*}$ be a prefix closed set of histories, $\sigma_{1}, \sigma_{2}, \tau_{1}, \tau_{2}$ strategies of Even and Odd in $G(s)$. We say that $\left(\sigma_{1}, \tau_{1}\right)$ and $\left(\sigma_{2}, \tau_{2}\right)$ are equivalent on $X$ if for each history $h=h^{\prime} s^{\prime} \in X$, if $s^{\prime} \in S_{0}$ then $\sigma_{1}\left(s^{\prime} \mid h^{\prime}\right)=\sigma_{2}\left(s^{\prime} \mid h^{\prime}\right)$ and if $s^{\prime} \in S_{1}$ then $\tau_{1}\left(s^{\prime} \mid h^{\prime}\right)=\tau_{2}\left(s^{\prime} \mid h^{\prime}\right)$.

We say that $\left(\sigma_{1}, \tau_{1}\right)$ and $\left(\sigma_{2}, \tau_{2}\right)$ are equivalent over a Borel set $U$ of plays if they are equivalent over the set of all prefixes of $U$.

The following lemma resumes elementary facts concerning the probabilities $P_{\sigma, \tau}$ that will be used frequently, most of the time tacitly, in the sequel.

Lemma 4. (1) If $\left(\sigma_{1}, \tau_{1}\right)$ and $\left(\sigma_{2}, \tau_{2}\right)$ are equivalent over a prefix closed set $X$ of histories then for each Borel set $U$ of plays in $G(s)$ such that all prefixes of $U$ belong to $X$ we have $P_{\sigma_{1}, \tau_{1}}(U)=P_{\sigma_{2}, \tau_{2}}(U)$.

(2) For all strategies $\sigma$ and $\tau$ and histories $h=h^{\prime} s^{\prime}$ in $G(s)$,

$$
P_{\sigma, \tau}\left(X \cap h S^{\omega}\right)=P_{\sigma, \tau}\left(h S^{\omega}\right) P_{\sigma_{h}, \tau_{h}}\left(s^{\prime}\left(h^{-1} X\right)\right),
$$

where $h^{-1} X=\{u \mid h u \in X\}$. 
Proof. (2) $P_{\sigma, \tau}\left(X \cap h S^{\omega}\right)=P_{\sigma, \tau}\left(X \cap h S^{\omega} \mid h S^{\omega}\right) P_{\sigma, \tau}\left(h S^{\omega}\right)$. However, $P_{\sigma, \tau}(X \cap$ $\left.h S^{\omega} \mid h S^{\omega}\right)$ is equal to the probability of winning the residual game with the residual strategies.

The proof of Proposition 2 goes through several lemmas. Let $\alpha$ and $\beta$ be strategies for a player $i$ in the games $G(s)$ and $G\left(s^{\prime}\right)$ respectively, $s, s^{\prime} \in S$. Then by $\alpha[\beta]$ we note a new strategy of $i$ in $G(s)$ that informally can be described as follows: play as $\alpha$ till the first visit to $s^{\prime}$, next play always according to $\beta$. Formally, if $h \in s S^{*}$ and the last state of $h$ belongs to $S_{i}$ then

$$
\alpha[\beta](h)= \begin{cases}\alpha(h) & \text { if } h \text { does not contain } s^{\prime} \\ \beta\left(s^{\prime} h^{\prime \prime}\right) & \text { if } h=h^{\prime} s^{\prime} h^{\prime \prime} \text { and } h^{\prime} \text { does not contain } s^{\prime} .\end{cases}
$$

Lemma 5. Let $G$ be a parity game. Let $H=s^{\prime}(S \backslash\{s\})^{*}$ be the set of histories that start at $s^{\prime}$ and never visit $s, E=s^{\prime}(S \backslash\{s\})^{\omega}$ the set of (infinite) plays that start at $s^{\prime}$ and never visit $s$ and $\bar{E}=s^{\prime} S^{\omega} \backslash E$ the set of plays that start at $s^{\prime}$ and visit at least once s. Let $\sigma^{\prime}, \tau^{\prime}$ be strategies of Even and Odd in $G\left(s^{\prime}\right)$. Then

(1) $P_{\sigma^{\prime}, \tau^{\prime}}\left(\operatorname{Win}_{0}\left(s^{\prime}\right)\right)=$

$\left.P_{\sigma^{\prime}, \tau^{\prime}}\left(\operatorname{Win}_{0}\left(s^{\prime}\right)\right) \cap E\right)+\sum_{h \in H} P_{\sigma^{\prime}, \tau^{\prime}}\left(h s S^{\omega}\right) P_{\sigma_{h s}^{\prime}, \tau_{h s}^{\prime}}\left(\operatorname{Win}_{0}(s)\right)$,

where $\sigma_{h s}^{\prime}$ and $\tau_{h s}^{\prime}$ are residual strategies.

(2) If $\sigma_{1}, \tau_{1}, \sigma_{2}, \tau_{2}$ are strategies in $G(s)$ such that

$P_{\sigma_{2}, \tau_{2}}\left(\operatorname{Win}_{0}(s)\right) \leq P_{\sigma_{1}, \tau_{1}}\left(\mathrm{Win}_{0}(s)\right)$ then

$P_{\sigma^{\prime}\left[\sigma_{2}\right], \tau^{\prime}\left[\tau_{2}\right]}\left(\operatorname{Win}_{0}\left(s^{\prime}\right)\right) \leq P_{\sigma^{\prime}\left[\sigma_{1}\right], \tau^{\prime}\left[\tau_{1}\right]}\left(\operatorname{Win}_{0}\left(s^{\prime}\right)\right)$.

Proof. Note that $\operatorname{Win}_{0}\left(s^{\prime}\right)=\left(\operatorname{Win}_{0}\left(s^{\prime}\right) \cap E\right) \cup\left(\operatorname{Win}_{0}\left(s^{\prime}\right) \cap \bar{E}\right)$ and $\bar{E}=$ $\bigcup_{h \in H} h s S^{\omega}$. By countable additivity of measures we get $P_{\sigma^{\prime}, \tau^{\prime}}\left(\operatorname{Win}_{0}\left(s^{\prime}\right)\right)=$ $P_{\sigma^{\prime}, \tau^{\prime}}\left(\operatorname{Win}_{0}\left(s^{\prime}\right) \cap E\right)+\sum_{h \in H} P_{\sigma^{\prime}, \tau^{\prime}}\left(\operatorname{Win}_{0}\left(s^{\prime}\right) \cap h s S^{\omega}\right)$. By Lemma 4 and since $s(h s)^{-1} \operatorname{Win}_{0}\left(s^{\prime}\right)=\operatorname{Win}_{0}(s), \quad P_{\sigma^{\prime}, \tau^{\prime}}\left(\operatorname{Win}_{0}\left(s^{\prime}\right) \cap h s S^{\omega}\right)=$ $\left.P_{\sigma^{\prime}, \tau^{\prime}}\left(h s S^{\omega}\right) P_{\sigma_{h s}^{\prime}, \tau_{h s}^{\prime}}\left(\operatorname{Win}_{0}(s)\right)\right)$.

Now applying two times (1) (and Lemma 4 to substitute equivalent strategies) we get

$P_{\sigma^{\prime}\left[\sigma_{2}\right], \tau^{\prime}\left[\tau_{2}\right]}\left(\operatorname{Win}_{0}\left(s^{\prime}\right)\right)=$

$\left.P_{\sigma^{\prime}, \tau^{\prime}}\left(\operatorname{Win}_{0}\left(s^{\prime}\right)\right) \cap E\right)+\sum_{h \in H} P_{\sigma^{\prime}, \tau^{\prime}}\left(h s S^{\omega}\right) P_{\sigma_{2}, \tau_{2}}\left(\operatorname{Win}_{0}(s)\right) \leq$

$\left.P_{\sigma^{\prime}, \tau^{\prime}}\left(\operatorname{Win}_{0}\left(s^{\prime}\right)\right) \cap E\right)+\sum_{h \in H} P_{\sigma^{\prime}, \tau^{\prime}}\left(h s S^{\omega}\right) P_{\sigma_{1}, \tau_{1}}\left(\operatorname{Win}_{0}(s)\right)=$ $P_{\sigma^{\prime}\left[\sigma_{1}\right], \tau^{\prime}\left[\tau_{1}\right]}\left(\operatorname{Win}_{0}\left(s^{\prime}\right)\right)$.

Lemma 6. Let $s$ and $s^{\prime}$ be two distinct states of a parity game $G$ such that $G(s)$ and $G\left(s^{\prime}\right)$ have values. We suppose that $\sigma, \bar{\sigma}, \bar{\sigma}^{\prime}$ are respectively any strategy in $G(s)$, an optimal strategy in $G(s)$ and an optimal strategy in $G\left(s^{\prime}\right)$ for the player Even. Similarly, $\tau, \bar{\tau}, \bar{\tau}^{\prime}$ are respectively any strategy in $G(s)$, an optimal strategy in $G(s)$ and an optimal strategy in $G\left(s^{\prime}\right)$ for player Odd. Then the following holds:

(i) $P_{\sigma, \tau\left[\bar{\tau}^{\prime}\right]}\left(\operatorname{Win}_{0}(s)\right) \leq P_{\sigma\left[\bar{\sigma}^{\prime}\right], \tau\left[\bar{\tau}^{\prime}\right]}\left(\operatorname{Win}_{0}(s)\right) \leq P_{\sigma\left[\bar{\sigma}^{\prime}\right], \tau}\left(\operatorname{Win}_{0}(s)\right)$.

(ii) The strategies $\bar{\sigma}\left[\bar{\sigma}^{\prime}\right]$ and $\bar{\tau}\left[\bar{\tau}^{\prime}\right]$ are optimal for players Even and Odd respectively in the game $G(s)$. 
Proof. Under the notation of Lemma 5 we get

$$
\begin{aligned}
P_{\sigma, \tau\left[\bar{\tau}^{\prime}\right]}\left(\operatorname{Win}_{0}(s)\right)=P_{\sigma, \tau\left[\bar{\tau}^{\prime}\right]}\left(\operatorname{Win}_{0}(s) \cap E\right) \cup \\
\sum_{h \in H} P_{\sigma, \tau\left[\bar{\tau}^{\prime}\right]}\left(h s^{\prime} S^{\omega}\right) P_{\sigma h s^{\prime}, \bar{\tau}^{\prime}}\left(\operatorname{Win}_{0}\left(s^{\prime}\right)\right),
\end{aligned}
$$

since the residual strategy $\left(\tau\left[\bar{\tau}^{\prime}\right]\right)_{h s^{\prime}}$ is equal $\bar{\tau}^{\prime}$.

But by optimality of the strategies $\bar{\sigma}^{\prime}$ and $\bar{\tau}^{\prime}$ :

$P_{\sigma_{h s^{\prime}}, \bar{\tau}^{\prime}}\left(\operatorname{Win}_{0}\left(s^{\prime}\right)\right) \leq \operatorname{val}_{0}\left(G\left(s^{\prime}\right)\right)=P_{\bar{\sigma}^{\prime}, \bar{\tau}^{\prime}}\left(\operatorname{Win}_{0}\left(s^{\prime}\right)\right)$. Putting this into (2) and applying again Lemma [5] get the first inequality in (ii). The second inequality in (i) is symmetrical.

To prove (iil) note the following sequence of relations with $\tau$ ranging over all strategies of Odd in $G(s): \operatorname{val}_{0}(G(s))=\inf _{\tau} P_{\bar{\sigma}, \tau}\left(\operatorname{Win}_{0}(s)\right) \leq$

$\inf _{\tau} P_{\bar{\sigma}, \tau\left[\bar{\tau}^{\prime}\right]}\left(\operatorname{Win}_{0}(s)\right) \leq \inf _{\tau} P_{\bar{\sigma}\left[\bar{\sigma}^{\prime}\right], \tau}\left(\operatorname{Win}_{0}(s)\right)$, where the first equality follows from the optimality of $\bar{\sigma}$, the next inequality follows from the fact that if $\tau$ ranges over all strategies of Odd in $G(s)$ then $\tau\left[\bar{\tau}^{\prime}\right]$ ranges over some subset of his strategies and the last inequality follows from (ii) with $\bar{\sigma}$ replacing $\sigma$. However, this means that $\bar{\sigma}\left[\bar{\sigma}^{\prime}\right]$ is optimal for the player Even. The optimality of $\bar{\tau}\left[\bar{\tau}^{\prime}\right]$ follows by symmetry.

Lemma 7. Under Conditions $A$ and $B$, $\operatorname{val}_{0}\left(G_{2}(s)\right) \leq \operatorname{val}_{0}\left(G_{1}(s)\right)$ if and only if for all states $s^{\prime}$, $\operatorname{val}_{0}\left(G_{2}\left(s^{\prime}\right)\right) \leq \operatorname{val}_{0}\left(G_{1}\left(s^{\prime}\right)\right)$.

Proof. Let $\bar{\sigma}_{2}^{\prime}, \bar{\sigma}_{2}, \bar{\sigma}_{1}^{\prime}, \bar{\sigma}_{1}$ be optimal strategies of player Even in the games $G_{2}\left(s^{\prime}\right), G_{2}(s), G_{1}\left(s^{\prime}\right), G_{1}(s)$ respectively. Similarly, $\bar{\tau}_{2}^{\prime}, \bar{\tau}_{2}, \bar{\tau}_{1}^{\prime}, \bar{\tau}_{1}$ are optimal in the same games for Odd.

From Lemma 6] we know that $\bar{\sigma}_{2}^{\prime}\left[\bar{\sigma}_{2}\right]$ is optimal in $G_{2}\left(s^{\prime}\right)$ for Even. On the other hand, $\bar{\tau}_{1}^{\prime}\left[\bar{\tau}_{2}\right]$ can be seen as a strategy of Odd in $G_{2}\left(s^{\prime}\right)$ since until the first visit to $s$ playing in $G_{1}$ or in $G_{2}$ is the same. These remarks imply that $\operatorname{val}_{0}\left(G_{2}\left(s^{\prime}\right)\right) \leq P_{\bar{\sigma}_{2}^{\prime}\left[\bar{\sigma}_{2}\right], \bar{\tau}_{1}^{\prime}\left[\bar{\tau}_{2}\right]}\left(\operatorname{Win}_{0}\left(s^{\prime}\right)\right)$. On the other hand, $P_{\bar{\sigma}_{2}, \bar{\tau}_{2}}\left(\operatorname{Win}_{0}(s)\right)=$ $\operatorname{val}_{0}\left(G_{2}(s)\right) \leq \operatorname{val}_{0}\left(G_{1}(s)\right)=P_{\bar{\sigma}_{1}, \bar{\tau}_{1}}\left(W_{\sigma_{0}}(s)\right)$ implies, by Lemma 5 ,

$P_{\bar{\sigma}_{2}^{\prime}\left[\bar{\sigma}_{2}\right], \bar{\tau}_{1}^{\prime}\left[\bar{\tau}_{2}\right]}\left(\operatorname{Win}_{0}\left(s^{\prime}\right)\right) \leq P_{\bar{\sigma}_{2}^{\prime}\left[\bar{\sigma}_{1}\right], \bar{\tau}_{1}^{\prime}\left[\bar{\tau}_{1}\right]}\left(\operatorname{Win}_{0}\left(s^{\prime}\right)\right)$. But by optimality of $\bar{\tau}_{1}^{\prime}\left[\bar{\tau}_{1}\right]$ the right hand side of the last inequality is not greater than $\operatorname{val}_{0}\left(G_{1}\left(s^{\prime}\right)\right)$.

Lemma 8. Under Conditions $A$ and $B$, if $s \in \mathrm{V}_{1}^{>0}\left(G_{1}\right) \cap \mathrm{V}_{1}^{>0}\left(G_{2}\right)$ then $\mathrm{V}_{1}^{>0}\left(G_{1}\right)=\mathrm{V}_{1}^{>0}\left(G_{2}\right)$.

Proof. W.l.o.g. we can assume that $0<\operatorname{val}_{1}\left(G_{1}(s)\right) \leq \operatorname{val}_{1}\left(G_{2}(s)\right)$. Then by Lemma [7, since $\operatorname{val}_{1}=1-\operatorname{val}_{0}$, we get for all states $s^{\prime}, \operatorname{val}_{1}\left(G_{1}\left(s^{\prime}\right)\right) \leq$ $\operatorname{val}_{1}\left(G_{2}\left(s^{\prime}\right)\right)$, i.e. $\mathrm{V}_{1}^{>0}\left(G_{1}\right) \subseteq \mathrm{V}_{1}^{>0}\left(G_{2}\right)$. To show the inverse inclusion take $s^{\prime}$ such that

$\operatorname{val}_{1}\left(G_{2}\left(s^{\prime}\right)\right)>0$. Let $\bar{\tau}_{2}^{\prime}$ be optimal for Odd in the game $G_{2}\left(s^{\prime}\right)$ and $\bar{\tau}_{1}$ optimal for Odd in $G_{1}(s)$. Note that $\bar{\tau}_{2}^{\prime}\left[\bar{\tau}_{1}\right]$ is in fact a strategy in the game $G_{1}\left(s^{\prime}\right)$ since once the state $s$ is visited player Odd begins to use $\bar{\tau}_{1}$.

Suppose that Odd plays according to the strategy $\bar{\tau}_{2}^{\prime}\left[\bar{\tau}_{1}\right]$ against some strategy $\sigma$. If with the probability 1 the token never visits $s$ then in fact Odd plays always according to $\bar{\tau}_{2}^{\prime}$ and therefore wins with a positive probability. 
If the token visits $s$ with some positive probability then Odd plays from this moment onwards according to $\bar{\tau}_{1}$ and again wins with a positive probability. We can conclude that Odd has a strategy in $G_{1}\left(s^{\prime}\right)$ to win with a positive probability. (The reasoning can be formalized easily with the help of Lemma 5).

In the sequel we assume Condition A, i.e. $s$ has two successors: $s_{1}$ and $s_{2}$.

Let $\Pi_{k}, k=1,2$, be mappings acting on histories $h \in s S^{*}(S \backslash\{s\})$ and defined as

$$
\Pi_{k}(h)= \begin{cases}h & \text { if } h=s s_{k} h^{\prime} \text { for some } h^{\prime} \in S^{*} \\ 1 & \text { otherwise. }\end{cases}
$$

Let $h \in s S^{*}(S \backslash\{s\})$ be a history in $G(s)$ ending in a state different from $s$. The loop factorization of $h$ is the unique factorization of the form $h=h_{0} h_{1} \ldots h_{l}$, where each factor $h_{i}, 1 \leq i \leq l$, is of the form $h_{i}=s h_{i}^{\prime}$ and $h_{i}^{\prime}$ do not contain any occurrences of $s$. Since the factors $h_{i}$ do not end in $s$ and $s$ has exactly two successors $s_{1}$ and $s_{2}$, each $h_{i}$ begins either with $s s_{1}$ or $s s_{2}$.

Then for $k=1,2$, we set $\pi_{k}(h)=\Pi_{k}\left(h_{0}\right) \Pi_{k}\left(h_{1}\right) \ldots \Pi_{k}\left(h_{l}\right)$. Thus $\pi_{1}$ preserves the factors $h_{i}$ of $h$ that begin with $s s_{1}$ and erases the factors beginning with $s s_{2}$, the action of $\pi_{2}$ is symmetrical.

What is essential to observe is that $\pi_{1}(h)$ and $\pi_{2}(h)$ are histories in $G_{1}(s)$ and $G_{2}(s)$ respectively, in some sense $\pi_{k}$ erases the loops in the history $h$ that are incompatible with the game $G_{k}, k=1,2$.

Suppose that the Condition $\mathrm{A}$ is satisfied and $\tau_{1}$ and $\tau_{2}$ are strategies of player Odd in the games $G_{1}(s)$ and $G_{2}(s)$ respectively. We define an interleaving strategy $\tau=\tau_{1} \prec \tau_{2}$ of Odd in $G(s)$ in the following way. Let $h=h_{0} h_{1} \ldots h_{l}$ be the loop factorization of the history $h \in s S^{*}$ ending in a state of $S_{1}$.

Then we set

$$
\tau(h)= \begin{cases}\tau_{1}\left(\pi_{1}(h)\right) & \text { if the last factor } h_{l} \text { of } h \text { begins with } s s_{1} \\ \tau_{2}\left(\pi_{2}(h)\right) & \text { if the last factor } h_{l} \text { of } h \text { begins with } s s_{2}\end{cases}
$$

Intuitively, if during the last visit to $s$ player Even has chosen to go to $s_{1}$, as in the game $G_{1}$, then until the next visit to $s$ player Odd uses the strategy $\tau_{1}$ with the history appropriately adjusted to $\pi_{1}(h)$, otherwise he uses the strategy $\tau_{2}$ with the history $\pi_{2}(h)$.

In the sequel the mappings $\pi_{1}$ and $\pi_{2}$ will also be applied to infinite plays $h \in(s S)^{\omega}$ beginning at $s$. Again we factorize $h=h_{0} h_{1} \ldots$ where $h_{i}$ begin with $s$ and do not contain other occurrences of $s$. (Note that either we visit $s$ infinitely often and then $h$ has infinitely many factors or the last factor is infinite.) Next we erase some factors in the way indicated above with the mapping $\Pi_{k}$, $\pi_{k}(h)=\Pi_{k}\left(h_{0}\right) \Pi_{k}\left(h_{1}\right) \ldots$ If infinitely many factors begin with $s s_{k}$ or $s$ is visited finitely often and the last infinite factor begins with $s s_{k}$ then $\pi_{k}(h)$ is a play in $G_{k}(s), k=1,2$.

Lemma 9. Suppose that Conditions $A$ and $B$ hold and $s \in \mathrm{V}_{1}^{>0}\left(G_{1}\right) \cap \mathrm{V}_{1}^{>0}\left(G_{2}\right)$. If $\tau_{1}, \tau_{2}$ are persistent strategies of Odd in $G_{1}(s), G_{2}(s)$ over the sets $\mathrm{V}_{1}^{>0}\left(G_{1}\right)$ 
and $\mathrm{V}_{1}^{>0}\left(G_{2}\right)$ respectively then the interleaving strategy $\tau_{1}<\tau_{2}$ is persistent for the player Odd over $\mathrm{V}_{1}^{>0}\left(G_{1}\right)=\mathrm{V}_{1}^{>0}\left(G_{2}\right)$ in the game $G(s)$.

Proof. Set $U=\mathrm{V}_{1}^{>0}\left(G_{1}\right)=\mathrm{V}_{1}^{>0}\left(G_{2}\right)$ (see Lemma 8 ). The set of plays $s U^{\omega}$ can be partitioned into three sets: the set $A_{1}$ of plays that from some moment onwards do not take any more the edge $\left(s, s_{2}\right)$, the symmetrical set $A_{2}$ of plays that from some moment onwards do not take the edge $\left(s, s_{2}\right)$ and the set $B$ of plays that take infinitely many times $\left(s, s_{1}\right)$ as well as $\left(s, s_{2}\right)$. In $A_{1}$ from some moment onwards player Odd plays always the same strategy $\tau_{1}$, which is persistent thus he wins almost surely when staying in $A_{1}$. Similarly, he wins almost surely in $A_{2}$.

Playing in $B$, Odd switches infinitely often between strategies $\tau_{1}$ and $\tau_{2}$. Take $u \in B$ and forget for a moment the intervals when he plays according to $\tau_{2}$, i.e. consider $\pi_{1}(u)$. Over $\pi_{1}(u)$ he plays always according to $\tau_{1}$ thus almost surely the maximal color visited infinitely often in $\pi_{1}(u)$ is odd (by persistence of $\tau_{1}$ ). Symmetrically almost surely the maximal colour visited infinitely often in $\pi_{2}(u)$ is also odd. Thus the maximal colour visited infinitely often in $u$ should be odd as well almost surely. (This informal reasoning can be formalized by noting that for any Borel subset $K$ of plays in $G_{1}(s)$ that stay in $U, P_{\sigma, \tau}\left(\pi_{1}^{-1}(K) \cap B\right) \leq$ $\sup _{\sigma_{1}} P_{\sigma_{1}, \tau_{1}}(K)$, where $\sigma_{1}$ ranges over strategies of Even in $G_{1}(s)$ and similar property holds in $G_{2}$ ).

Lemma 10. Suppose that $G$ is a game having values for all starting positions, $s \in \mathrm{V}_{1}^{>0}(G)$ and $\bar{\tau}$ a residually optimal strategy of Odd in $G(s)$ which is also persistent on $\mathrm{V}_{1}^{>0}(G)$. Let $\sigma$ be any strategy of Even in $G(s)$. Set $X=\mathrm{V}_{0}^{=1}(G)=$ $S \backslash \mathrm{V}_{1}^{>0}(G)$. Let $A=s(S \backslash\{s\})^{*} X S^{\omega}$ be the set of plays that start at $s$ and hit eventually $X$ without any other visit to $s$ in the meantime than the first one, $B=s(S \backslash X)^{*} s S^{\omega}$ the set of plays that return to $s$ without hitting $X, a=P_{\sigma, \bar{\tau}}(A)$, $b=P_{\sigma, \bar{\tau}}(B)$. Then $\operatorname{val}_{0}(G(s)) \geq \frac{a}{1-b}$.

Proof. The set of plays starting at $s$ can be partitioned into three disjoint sets $A$, $B$ and the set $C$ of plays that after leaving $s$ never return to $s$ and never hit $X$. By the persistence of $\bar{\tau}$ we get that $P_{\sigma, \bar{\tau}}\left(C \cap \operatorname{Win}_{1}(s)\right)=P_{\sigma, \bar{\tau}}(C)$, i.e. $P_{\sigma, \bar{\tau}}(C \cap$ $\left.\operatorname{Win}_{0}(s)\right)=0$. On the other hand, since $B$ is just a disjoint union of the sets of the form $h S^{\omega}$, where $H$ consists of the histories of the form $s h^{\prime} s$ where $h^{\prime}$ does not contain neither $s$ nor elements of $X$, by Lemma 4 we get $P_{\sigma, \bar{\tau}}\left(\operatorname{Win}_{0}(s) \cap B\right)=$ $\sum_{h \in H} P_{\sigma, \bar{\tau}}\left(h S^{\omega}\right) P_{\sigma_{h}, \bar{\tau}_{h}}\left(\operatorname{Win}_{0}(s)\right)$. But $P_{\sigma_{h}, \bar{\tau}_{h}}\left(\operatorname{Win}_{0}(s)\right) \leq \operatorname{val}_{0}(G(s))$ since $\bar{\tau}_{h}$ is optimal (because $\bar{\tau}$ is residually optimal). Summarizing all these identities we get $P_{\sigma, \bar{\tau}}\left(\operatorname{Win}_{0}(s)\right)=P_{\sigma, \bar{\tau}}\left(A \cap \operatorname{Win}_{0}(s)\right)+P_{\sigma, \bar{\tau}}\left(B \cap \operatorname{Win}_{0}(s)\right)+P_{\sigma, \bar{\tau}}\left(C \cap \operatorname{Win}_{0}(s)\right) \leq$ $P_{\sigma, \bar{\tau}}(A)+\sum_{h \in H} P_{\sigma, \bar{\tau}}\left(h S^{\omega}\right) P_{\sigma_{h}, \bar{\tau}_{h}}\left(\operatorname{Win}_{0}(s)\right) \leq a+\operatorname{val}_{0}(G(s)) \cdot \sum_{h \in H} P_{\sigma, \bar{\tau}}\left(h S^{\omega}\right)=$ $a+b \cdot \operatorname{val}_{0}(G(s))$. Since this inequality is valid for all strategies of Even we get $\operatorname{val}_{0}(G(s))=\sup _{\sigma} P_{\sigma, \bar{\tau}}\left(\operatorname{Win}_{0}(s)\right) \leq a+b \cdot \operatorname{val}_{0}(G(s))$.

\section{Proof of Proposition 2}

(i) was already proved in Lemma 7 (ii) is just a consequence of (iii) and (iv). (iii) is obvious, if player Even takes always the transition $s \longrightarrow s_{1}$ when visiting 
$s$ in $G$ then from the point of view of Odd playing against such a strategy is like playing in $G_{1}$, thus certainly he cannot win more than he wins in $G_{1}$.

To prove (iv) we consider two cases.

Set $Y=\mathrm{V}_{1}^{>0}\left(G_{1}\right)=\mathrm{V}_{1}^{>0}\left(G_{1}\right) \cap \mathrm{V}_{1}^{>0}\left(G_{2}\right)$, where the last equality follows from (i).

If $s \notin Y$ then for each $s^{\prime} \in Y$ it suffices to take as $\bar{\tau}$ the strategy $\bar{\tau}_{1}$ assumed in Condition D. Player Odd can use it as long as the token stays in $Y$ just as in the game $G_{1}\left(s^{\prime}\right)$. This strategy secures for him the winning probability of at least $\operatorname{val}_{1}\left(G_{1}(s)\right)$, is residually optimal and is persistent on $Y$.

Thus the only interesting case is when $s \in Y$. Then, by Lemma 8$] Y=$ $\mathrm{V}_{1}^{>0}\left(G_{1}\right)=\mathrm{V}_{1}^{>0}\left(G_{2}\right)$. Suppose that $\bar{\tau}_{1}$ and $\bar{\tau}_{2}$ are residually optimal persistent strategies of Odd in $G_{1}(s)$ and $G_{2}(s)$. Set $\bar{\tau}=\bar{\tau}_{1} \prec \bar{\tau}_{2}$. We prove first that $\bar{\tau}$ has all required properties in the game $G(s)$.

The persistence of $\bar{\tau}$ follows from Lemma 9 To prove the optimality of $\bar{\tau}$ take any strategy $\sigma$ in $G(s)$. Set $t=\operatorname{val}_{0}\left(G_{2}(s)\right)=\min \left\{\operatorname{val}_{0}\left(G_{1}(s)\right)\right.$, $\left.\operatorname{val}_{0}\left(G_{2}(s)\right)\right\}$. Thus our aim is to prove that $P_{\sigma, \bar{\tau}}\left(\operatorname{Win}_{0}(s)\right) \leq t$.

By the persistence of $\bar{\tau}, P_{\sigma, \bar{\tau}}\left(\operatorname{Win}_{0}(s)\right)=P_{\sigma, \bar{\tau}}\left(\operatorname{Win}_{0}(s) \cap D\right)$, where $D$ is the set of plays that hit eventually the set $X=S \backslash Y$ of states. But $P_{\sigma, \bar{\tau}}\left(\operatorname{Win}_{0}(s) \cap\right.$ $D) \leq P_{\sigma, \bar{\tau}}(D)$. Therefore in the sequel it suffices to prove that $P_{\sigma, \bar{\tau}}(D) \leq t$.

Note that $D$ is a disjoint union of the sets $u S^{\omega}, u \in U$, where $U$ consists of histories of the form $u=s u^{\prime} x$ with $x \in X$ and $u^{\prime}$ not containing elements of $X$.

Let $U_{n}=\{u \in U \mid$ the number of occurrences of $s$ in $u \leq n\}$.

Then $D=U S^{\omega}=\bigcup_{n \geq 1} U_{n} S^{\omega}$ and by measures monotonicity $P_{\sigma, \bar{\tau}}(D)=$ $\lim _{n} P_{\sigma, \bar{\tau}}\left(U_{n} S^{\omega}\right)$. Therefore to guarantee $P_{\sigma, \bar{\tau}}(D) \leq t$ it suffices to prove $P_{\sigma, \bar{\tau}}\left(U_{n} S^{\omega}\right) \leq t$ for all $n$.

We proceed by induction on $n$. The case $n=1$ is a special case of the induction step. Thus assume that $P_{\sigma, \bar{\tau}}\left(U_{n} S^{\omega}\right) \leq t$ and we sketch the proof of similar inequality for $n+1$. By Bayes formula:

$$
\begin{aligned}
& P_{\sigma, \bar{\tau}}\left(U_{n+1} S^{\omega}\right)= \\
& P_{\sigma, \bar{\tau}}\left(U_{n+1} S^{\omega} \mid s s_{1} S^{\omega}\right) P_{\sigma, \bar{\tau}}\left(s s_{1} S^{\omega}\right)+P_{\sigma, \bar{\tau}}\left(U_{n+1} S^{\omega} \mid s s_{2} S^{\omega}\right) P_{\sigma, \bar{\tau}}\left(s s_{2} S^{\omega}\right) \\
& \quad=P_{\sigma, \bar{\tau}}\left(U_{n+1} S^{\omega} \mid s s_{1} S^{\omega}\right) \sigma\left(s_{1} \mid s\right)+P_{\sigma, \bar{\tau}}\left(U_{n+1} S^{\omega} \mid s s_{2} S^{\omega}\right) \sigma\left(s_{2} \mid s\right)
\end{aligned}
$$

Let $\sigma_{1}, \sigma_{2}$ be strategies identical with $\sigma$ everywhere except on the first step: $\sigma_{1}\left(s_{1} \mid s\right)=\sigma_{2}\left(s_{2} \mid s\right)=1, \sigma_{1}\left(s_{2} \mid s\right)=\sigma_{2}\left(s_{1} \mid s\right)=0$. Then we can replace the conditional probabilities in (3) since

$$
\begin{aligned}
& P_{\sigma, \bar{\tau}}\left(U_{n+1} S^{\omega} \mid s s_{1} S^{\omega}\right)=P_{\sigma_{1}, \bar{\tau}}\left(U_{n+1} S^{\omega}\right) \text { and } \\
& \qquad P_{\sigma, \bar{\tau}}\left(U_{n+1} S^{\omega} \mid s s_{2} S^{\omega}\right)=P_{\sigma_{2}, \bar{\tau}}\left(U_{n+1} S^{\omega}\right) .
\end{aligned}
$$

Now note that $P_{\sigma_{1}, \bar{\tau}}\left(U_{n+1} S^{\omega}\right)$ decomposes into the sum of probabilities to hit $X$ without returning to $s$ - we note this probability $a_{1}$ in the notation of Lemma [10] - and the probability to return to $s$ without hitting $X$ - that we note $b_{1}-$ multiplied by the probability to visit afterwards $s$ at most $n$ times before hitting $X$, which is less or equal $t$ by the induction hypothesis. In other words, 
$P_{\sigma_{1}, \bar{\tau}}\left(U_{n+1} S^{\omega}\right) \leq a_{1}+b_{1} \cdot t$, where by Lemma $10 t \geq \operatorname{val}_{0}\left(G_{1}(s)\right) \geq \frac{a_{1}}{1-b_{1}}$. Similar inequalities hold for $P_{\sigma_{2}, \bar{\tau}}\left(U_{n+1} S^{\omega}\right)$. These inequalities and Eqs (3) and (4) imply that $P_{\sigma, \bar{\tau}}\left(U_{n+1} S^{\omega}\right) \leq t \cdot\left(\sigma\left(s_{1} \mid s\right)+\sigma\left(s_{2} \mid s\right)\right)=t$.

The proof that $\bar{\tau}$ is not only optimal but also residually optimal follows a similar reasoning.

For plays starting in the states $s^{\prime}$ other than $s$ we can take as a strategy in $G\left(s^{\prime}\right)$ the strategy $\bar{\tau}_{1}^{\prime}[\bar{\tau}]$ where $\bar{\tau}_{1}^{\prime}$ is optimal and persistent in $G_{1}\left(s^{\prime}\right)$ and $\bar{\tau}$ is the interleaving strategy in $G(s)$ for which we have just proved the optimality.

Acknowledgements. We thank an anonymous referee for pointing to us the reference [14.

\section{References}

1. A. Arnold and D. Niwiński. Rudiments of $\mu$-calculus, volume 146 of Studies in Logic and the Foundations of Mathematics. Elsevier, 1997.

2. K. Chatterejee, M. Jurdziński, and T.A. Henzinger. Quantitative stochastic parity games. In $S O D A, 2003$. to appear.

3. A. Condon. The complexity of stochastic games. Information and Computation, 96:203-224, 1992.

4. C. Courcoubetis and M. Yannakakis. Markov decision processes and regular events. In ICALP'90, volume 443 of LNCS, pages 336-349. Springer, 1990.

5. L. de Alfaro and T.A. Henzinger. Concurrent $\omega$-regular games. In LICS'00, pages 142-154. IEEE Computer Society Press, 2000.

6. L. de Alfaro, T.A. Henzinger, and O. Kupferman. Concurrent reachability games. In FOCS'98, pages 564-575. IEEE Computer Society Press, 1998.

7. L. de Alfaro and R. Majumdar. Quantitative solution to omega-regular games. In STOC'01, pages 675-683. ACM Press, 2001.

8. Luca de Alfaro. Formal Verification of Probabilistic Systems. PhD thesis, Stanford University, december 1997.

9. E.A. Emerson and C. Jutla. Tree automata, $\mu$-calculus and determinacy. In FOCS'91, pages 368-377. IEEE Computer Society Press, 1991.

10. E.A. Emerson, C.S. Jutla, and A.P. Sistla. On model checking of fragments of $\mu$-calculus. In $C A V^{\prime} 93$, volume 697 of $L N C S$, pages 385-396. Springer, 1993.

11. J. Filar and K. Vrieze. Competitive Markov Decision Processes. Springer, 1997.

12. Hugo Gimbert, September 2003. personal communication.

13. D.A. Martin. The determinacy of Blackwell games. Journal of Symbolic Logic, 63(4):1565-1581, 1998.

14. A.K. McIver and C.C. Morgan. Games, probability and the quantitative $\mu$-calculus qmu. In Proc. LPAR, volume 2514 of LNAI, pages 292-310. Springer, 2002. full version arxiv.org/abs/cs.LO/0309024.

15. L. S. Shapley. Stochastic games. Proceedings Nat. Acad. of Science USA, 39:10951100, 1953. 